\title{
BMJ Open Effects of adding adjunctive hyperbaric oxygen therapy to standard wound care for diabetic foot ulcers: a protocol for a systematic review with meta-analysis and trial sequential analysis
}

To cite: Vinkel J, Holm NFR, Jakobsen JC, et al. Effects of adding adjunctive hyperbaric oxygen therapy to standard wound care for diabetic foot ulcers: a protocol for a systematic review with meta-analysis and trial sequential analysis. BMJ Open 2020;10:e031708. doi:10.1136/ bmjopen-2019-031708

- Prepublication history and additional material for this paper are available online. To view these files, please visit the journal online (http://dx.doi. org/10.1136/bmjopen-2019031708).

Received 03 June 2019 Revised 10 February 2020 Accepted 06 March 2020
Check for updates

(C) Author(s) (or their employer(s)) 2020. Re-use permitted under CC BY-NC. No commercial re-use. See rights and permissions. Published by BMJ.

For numbered affiliations see end of article.

Correspondence to

Dr Julie Vinkel;

julie.vinkel.clausen@regionh.dk

\section{ABSTRACT}

Introduction Diabetic foot ulcer represents a major health problem globally. Preliminary studies have indicated that systemic treatment of diabetic foot ulcer patients with hyperbaric oxygen therapy have beneficial effects on wound healing, risk of amputation, glycaemic control, atherosclerosis, inflammatory markers and other clinical and laboratory parameters. This protocol for a systematic review aims at identifying the beneficial and harmful effects of adding hyperbaric oxygen therapy to standard wound care for diabetic foot ulcers.

Methods and analysis This protocol was performed following the recommendations of the Cochrane Collaboration and the eight-step assessment procedure suggested by Jakobsen and colleagues. We plan to include all relevant randomised clinical trials assessing the effects of hyperbaric oxygen therapy in the treatment of diabetic foot ulcer versus any control group with any intervention defined as standard wound care or similar, together with sham interventions. Our primary outcome will be: all-cause mortality, serious adverse events and quality of life. Our secondary outcomes will be: healing of index wound, major amputation and wound infection. Any eligible trial will be assessed and classified as either high risk of bias or low risk of bias, and our conclusions will be based on trials with low risk of bias. The analyses of the extracted data will be performed using Review Manager 5 and Trial Sequential Analysis. For both our primary and secondary outcomes, we will create a 'Summary of Findings' table and use GRADE (Grading of Recommendations Assessment, Development and Evaluation) assessment to assess the quality of the evidence.

Ethics and dissemination We use publicly accessible documents as evidence, there is no participant involvement at an individual level and an institutional ethics approval is not required. The results of the review will be sought published in a peer-reviewed journals, also in the event of insignificant results or null results, and thereby it will be disseminated to clinicians and public available.

PROSPERO registration number CRD42019139256.
Strengths and limitations of this study

- Application of an eight-step procedure for better validation of meta-analytical results in systematic reviews as suggested by Jakobsen et al.

- The predefined methodology is based on the Cochrane Handbook for systematic reviews of interventions.

- Control of type I errors and type II errors with trial sequential analysis.

- Multiple treatment elements are used and the treatment strategy may vary across the trials that we compare.

The review has six outcomes and several subgroup analyses, and thus many comparisons are performed. This statistical multiplicity increases the risk of type I error.

\section{INTRODUCTION}

Diabetic foot ulcer is a pathological condition that arises from diabetes and represents a major health problem throughout the world. ${ }^{1}$ The prevalence of diabetes and its consequences has been steadily increasing for the past three decades, and the WHO estimates a prevalence of 64 million persons with diabetes in the European region. ${ }^{2}$ The annual population-based incidence of diabetic foot ulcers ranges from $1.0 \%$ to $4.1 \% .^{3}$ It is estimated that $7.4 \%$ of people with diabetes in the UK have a history of active or previous foot ulcer, with a lifetime risk for developing a foot ulcer of $15 \%$. $^{34}$

Diabetic foot ulcer is defined as a foot affected by ulceration that is associated with neuropathy and peripheral arterial disease of the lower limb in a person with diabetes. ${ }^{5}$ Diabetic foot ulcers can be asymptomatic due to diabetic neuropathy, even in the presence of severe tissue loss. ${ }^{6}$ Neuropathy impedes 
pain impulses to the brain and leads to poor skin sensitivity. The condition will gradually worsen if/as the person continues to walk on the insensitive foot. ${ }^{7}$ As the condition aggravates and penetrates deeper layers of the skin, symptoms related to the neuropathy and ulceration may arise. Symptoms of neuropathy include burning sensation; pins and needles; shooting, sharp or stabbing pains and muscle cramps that are distributed symmetrically in both limbs. ${ }^{5}$ Neuropathic pain is associated with impaired sleep, high levels of anxiety and depression and may cause disability with need of help to walk (crutches or wheelchair)..$^{8-11}$ The symptoms of foot ulceration can be swelling, warmth around the wound, foul-smelling discharge seeping from the wound, as well as pain and firmness when the wound is touched.

Eventually the ulceration can be subject to microorganisms' invasion associated with inflammation including abscess formation, cellulitis, myositis, paronychia, necrotising fasciitis, septic arthritis, tendonitis and osteomyelitis by spreading to the bone after the establishment of infection. ${ }^{12} 13$ The incidence of amputation among people with diabetic foot ulcers depends on definition of amputation, local clinical practice as well as cultural, geographical and economic factors and therefore ranges between $8.3 \%$ to $28.6 \%$ in the literature. ${ }^{41415}$ Likewise, the physical, emotional and psychological consequences of amputation vary among populations and is dependent on the level of amputation as well as on social and cultural factors. ${ }^{1617}$

\section{Healthcare costs}

Diabetic foot ulcers inflict significant healthcare costs on individuals and healthcare systems. ${ }^{18}$

A large multicentre prospective observational study including several European countries estimated the direct cost of healing one foot ulcer was $7147 €$, but if no healing was achieved in 12 months, the cost rose to 18790 $€ .^{19}$ When amputation was necessary the hospital cost was $24540 €$ (2005 prices). The projected European costs of treatment of diabetic foot ulcers was estimated to be 10 billion euros per year in the middle of the 2000s decade. ${ }^{19}$ Likewise, a retrospective study from the USA involving 883463 diabetic foot ulcer patients found that hospital costs per diabetic foot ulcer admission were US $\$ 8145$ to US\$11 290, depending on the cause of admission. The cost of diabetic foot ulcer care in the USA was estimated to be US $\$ 1.38$ billion per year (2010 prices). ${ }^{20}$

\section{Diagnosis}

Diabetic foot ulcer is a clinical diagnosis that involves assessing both the foot ulcer and the risk factors for development and progression of a foot ulcer. ${ }^{72}$ The International Working Group on the Diabetic Foot (IWGDF) has been publishing and updating international Practical Guidelines on diagnostics and management of foot problems in persons with diabetes since 1999. Overall, the diagnostic process includes evaluating the following: ${ }^{21}$
- Type of foot ulcer: most ulcers can be classified as neuropathic, ischaemic or neuro-ischaemic. This classification includes assessment of the vascular tree with symptom-directed history, palpation of foot pulses and measurement of distal blood pressures.

- Cause of foot ulcer: evaluation of ill-fitting shoes, footwear behaviour, foot deformities and calluses.

- Site and depth of foot ulcer: ulcers on the plantar surface of the foot or in areas overlying a bony deformity are most often neuropathic. The depth of the ulcer can be difficult to determine due to the presence of overlying callus or necrosis. Ulcers in the tips of the toes or the lateral border of the foot are most commonly ischaemic or neuro-ischaemic ulcers.

- Signs of infection: signs and symptoms of infection such as fever, pain, increased white blood cell count, presence of substantial tissue damage and development of an abscess. The risk of osteomyelitis should be determined and debridement should be performed.

\section{Classification}

There is no consensus among researchers or clinicians about a validated foot ulcer classification system. ${ }^{22-24}$ More than 10 diabetic foot ulcer classification systems have been described by researchers. ${ }^{25}$ Historically, the most widely used classification system for describing diabetic foot ulcers is the Meggitt-Wagner classification. The Meggitt-Wagner classification system is relatively simple and includes five classes that focusses on infection and affected tissue layers. ${ }^{5}$ Although widely used both clinically and scientifically, this classification system has been criticised for both purposes. ${ }^{26}$ Other classification systems include more variables such as wound area and measures of blood perfusion in the skin surrounding the foot ulcer. A system used in routine clinical practice should be simple enough to be remembered and easily applied, such as University of Texas System or the SINBAD (Site, Ischaemia, Neuropathy, Bacterial Infection and Depth) system. ${ }^{24}$ For scientific purpose, the IWGDF has developed a classification system PEDIS, that has also been shown to be correlated with the risk of amputation. ${ }^{24} 27-29$

\section{Pathophysiology}

The pathogenesis of diabetic foot ulcers is a triopathy related to the following diabetic complications; neuropathy, arteriopathy and a modified response to infection. ${ }^{30}$

Sensory neuropathy results in progressive loss of protective sensation, as well as loss of sensation of posture and vibration. This causes an abnormal walking pattern, which in turn gives rise to foot deformities, limited joint mobility, abnormal biomechanical loading of the foot, thickened skin, subcutaneous haemorrhage and, ultimately, ulceration. Ulceration can also be caused by minor trauma caused by ill-fitting shoes or injuries, which persons with diabetes are predisposed to due to sensory neuropathy. ${ }^{7}$ Autonomic neuropathy leads to loss of sympathetic innervation, which results in 
dry skin that is more susceptible to lesions and bacterial habitation. ${ }^{30}$

Peripheral vascular disease in persons with diabetes plays an important role in the pathogenesis of foot ulcers and is directly related to the lack of healing and risk of amputation. However, the interaction of factors is complicated. Despite a technically successful surgical revascularisation, diabetic foot ulcers are slow to heal and often recidivates. Only $60 \%$ of the diabetic foot ulcer patients achieve wound healing 1 year after revascularisation and $40 \%$ of them experience recurrences or a new foot ulcer within another 12 months. ${ }^{31} 32$ The pathophysiological mechanism is complex not only due to structural changes in the larger vessels, but also functional changes in the microcirculation. ${ }^{30}$

Persons with diabetes are at increased risk of infection, including skin infections, mycoses, pneumonia, sepsis, bone and joint infection and endocarditis. The risk of hospitalisation and/or infection-related death is almost twofold compared with an age- and sex-matched population. ${ }^{33}$ The increased risk of infection is associated with hyperglycaemia. ${ }^{33}$ Normal wound healing occurs in a regulated, coordinated and orderly sequence that requires cells, signalling molecules and extracellular matrix formation and maintenance. All these key ingredients have been shown to be altered with diabetes and diabetic wounds often remain in the inflammatory phase. $^{34}$

\section{Standard wound care of diabetic foot ulcers}

International guidelines have been published and regularly updated by the IWGDF since 1999. The guidelines consist of five documents related to foot complications in diabetes with the following topics: prevention, footwear and offloading, peripheral artery disease, foot infections and wound healing interventions. ${ }^{6} 13$ 35-37 The result is an evidence-based global consensus on prevention and management of foot problems in diabetes that should be used for developing local guidelines in each country adapted to local circumstance. ${ }^{21}$

In all countries, there should be at least three levels of foot-care management constituting a multidisciplinary foot care team. The first level consists of a general practitioner, a podiatrist and a diabetic nurse. The second level is a hospital setting with diabetologist, surgeon (general, orthopaedic or foot), vascular surgeon, endovascular interventionist, podiatrist and diabetic nurse, in collaboration with a shoemaker, orthotist or prosthetist. The third level is a foot centre that is specialised in diabetic foot care with multiple experts from several disciplines that acts as a tertiary reference centre. ${ }^{38}$

Standard wound care involves:

- Relief of pressure and protection of the ulcer: treatment elements could be offloading devices such as total contact cast, removable walker rendered irremovable, a removable device, shoe-modifications, temporary footwear, felted foam in combination with appropriate footwear, toe-spacers or orthoses. Patients may be instructed to limit standing and walking and to use crutches.

- Restoration of foot perfusion: this includes examination with devices such as ankle pressure, ankle brachial index, toe pressure or transcutaneous oxygen measurement. Urgent vascular imaging and revascularisation should be considered. Efforts to reduce cardiovascular risk (cessation of smoking, control of hypertension and dyslipidaemia, use of aspirin or clopidogrel) should be emphasised.

- Treatment of infection: treatment element could be cleansing, debridement of necrotic tissue and surrounding callus as well as empirical oral antibiotic therapy. In deep infection urgent surgical intervention to remove necrotic tissue and drain abscesses should be performed. The antibiotic regimen should be adjusted based on clinical response, culture and sensitivity results from the deepest tissue involved.

- Metabolic control and treatment of comorbidity: glycaemic control should be optimised, if necessary with insulin. Oedema or malnutrition should be treated if present.

- Local wound care: this includes frequent inspection of the ulcer with repeated debridement as needed, dressings to control excess exudation and maintain moist environment, negative pressure therapy to postoperative wounds and hyperbaric oxygen therapy (see below) in poorly healing wounds.

Successful efforts to prevent and treat foot complications depend on a well-organised team that integrates the various disciplines involved and uses a holistic approach in which the ulcer is seen as a sign of multiorgan disease. ${ }^{38}$

\section{Definition of the intervention}

The first article describing adjunctive use of hyperbaric oxygen therapy was written in 1879 by the French surgeon Fontaine, who believed that pressurised oxygen chambers helped in patient anaesthesia. ${ }^{39}$ In the $1950 \mathrm{~s}$ and $1960 \mathrm{~s}$ Churchill-Davidson and Boerema's work led to acknowledgement of the value of hyperbaric oxygen therapy in radiation therapy, certain anaerobic infections, carbon monoxide poisoning and cardiac surgery. ${ }^{40}$

Hyperbaric oxygen therapy consists of breathing $100 \%$ oxygen $(\mathrm{FiO} 2=1.0)$ under elevated pressure, which is defined as a minimum of 1.4 atmosphere absolute $(141.86 \mathrm{kPa})$ in a pressure chamber. ${ }^{41}$ The chamber can either be a multi-chamber with room for treating several patients simultaneously, typically sitting side by side along the hull of the chamber, or a mono-chamber, which can treat only one patient in a supine position.

The oxygen is delivered by either a hood or a mask, and most mono-chambers are compressed with pure oxygen. ${ }^{42} \mathrm{~A}$ treatment session is divided into a compression phase of typically 5 to 10 min duration. A treatment phase and a corresponding decompression phase of 5 to 10 min duration will be made during the ascension 
to atmospheric pressure depending on local procedures and requirements to medical personnel. ${ }^{41}$

\section{The physiological effect}

The immediate effect of hyperbaric oxygen therapy is a rise in the oxygen partial pressure in the blood, and the amount of physically dissolved oxygen increases to a level that is sufficient to support otherwise hypoxic tissue. A tissue's oxygen supply is driven by the haemoglobin bound oxygen and tissue perfusion. ${ }^{43}$ The oxygen partial pressure gradient between the blood and the tissue cells is the vector that determines the diffusion direction to regions with the greatest oxygen turnover. The driving force is a gradual decrease in the oxygen partial pressure gradient that occurs when oxygen is transported to tissue cell mitochondria by diffusion. Hyperbaric oxygen therapy increases the oxygen tension in ischaemic wounds under conditions of adequate arterial inflow. ${ }^{44-46}$ A sustainable effect of hyperbaric oxygen therapy on tissue oxygenation is obtained through formation of new vessels by neovascularisation. After a hyperbaric treatment session, the oxygen tension can only stay above baseline for hours. However, it is assumed that the intermittent period of hypoxia and hyperoxia in wounds initiates a cascade reaction that ultimately stimulates neovascularisation through, among other things, an increase in vascular endothelial growth factor. ${ }^{45}$

Adverse events from hyperbaric oxygen therapy are assumed to be low and self-limited when they do occur. ${ }^{478}$ Adverse events are: barotraumatic lesions, sinus squeeze, seizures (oxygen toxicity), myopia (oxygen toxicity), cataract, pulmonary dyspnoea (oxygen toxicity), claustrophobia, hypoglycaemic among patients with diabetes, dizziness and weakness. ${ }^{47} 49$ The overall per pressurising incidence is described as 400 to 721:100 000, of which middle ear barotrauma is the most frequent. ${ }^{47}{ }^{49}$ Hyperbaric oxygen therapy has in several studies been suggested to be cost-effective as an adjunctive therapy to standard wound care..$^{50} 51$

\section{The therapeutic protocol for treatment of diabetic foot ulcers}

In recent years international guidelines for adjuvant hyperbaric oxygen therapy of diabetic foot ulcers have been published. ${ }^{31}{ }^{52}$ These guidelines recommend that patients with diabetic foot ulcers with Wagner grade 3 or higher, that have not shown significant improvement after 30 days of standard wound care, should be offered adjunctive hyperbaric oxygen therapy. ${ }^{52}$ Moreover, diabetic foot ulcer patients with Wagner grade 3 or higher, who have newly received a surgical debridement of an infected foot, should be treated with acute postoperative hyperbaric oxygen therapy in addition to standard care. ${ }^{52}$

The therapeutic range in hyperbaric oxygen therapy wound healing protocols is between 2.0 to 2.5 atmosphere absolute. ${ }^{41} \mathrm{~A}$ frequently used treatment regimen in many hyperbaric centres is the administration of hyperbaric oxygen therapy five times a week for 90 min per session.
The total number of sessions varies based on the response of the wound to treatment and may comprise 40 or more sessions in total. ${ }^{53}$

\section{Systemic effects of hyperbaric oxygen therapy}

Diabetic foot ulcers are linked to an increased risk of death. A recent cohort study including 414523 people with diabetes found that individuals with a diabetic foot ulcer were three times more likely to die at any time compared with individuals with diabetes who did not have a diabetic foot ulcer. ${ }^{54}$

The association between diabetic foot ulcers and death could not be explained by other known major complications of diabetes, including myocardial infarction, congestive heart failure, peripheral vascular diseasearterial insufficiency, cerebrovascular accident and chronic kidney disease. ${ }^{54}$ In fact, when placed together in a fully adjusted model, these complications of diabetes explained $<30 \%$ of the association between diabetic foot ulcer and death, suggesting that there are major unknown risk factors associated with diabetic foot ulcers. ${ }^{54}$ Hyperbaric oxygen therapy is a systemic therapy and it has been shown to have beneficial effects on glycaemic control, atherosclerosis, inflammatory markers and other clinical and laboratory parameters in patients with diabetic foot ulcerations. ${ }^{55-58}$ Moreover, hyperbaric oxygen therapy has a protective effect on the heart, by preventing prolonged QT interval in diabetic patients with foot ulcers, ${ }^{59}$ which has been associated with increased survival, ${ }^{60}$ and through a vagotonic effect that improves cardiac neural regulation in diabetic individuals with foot complications. ${ }^{61} \mathrm{An}$ explanation for this effect may be that diabetes is strongly associated with reduced microcapillary tissue perfusion; tissue hypoxia and neuronal dysfunction, all of which are improved by microcapillary angiogenesis as induced by systemic hyperbaric oxygen therapy. ${ }^{62-64}$

\section{Why is it important to do this review?}

A preliminary search identified a total of 15 previous reviews assessing the effects of hyperbaric oxygen therapy for diabetic foot ulcers. Only two of the 15 reviews comparing adjunctive hyperbaric oxygen therapy with standard wound care reported data on all-cause mortality. The Canadian Programme for Assessment of Technology reported all-cause mortality as an adverse event and found that two randomised controlled trials and one observational study described death as a study outcome, with overall three occurrences in the hyperbaric oxygen therapy groups and seven occurrences in the control groups ${ }^{65}$ Later Ontario health assessment 2017 reported all-cause mortality as a primary outcome, including GRADE (Grading of Recommendations Assessment,Development and Evaluation) assessment. Based on one randomised controlled trial and one non-randomised controlled study, they found three deaths in the hyperbaric oxygen therapy groups and six deaths in the control groups. This difference was not significant and the quality 
of evidence was moderate according to the GRADE criteria. ${ }^{66}$

Ten reviews reported adverse events of the intervention as descriptive outcomes. ${ }^{48} 526567-73$ Two reviews reported adverse events as a primary outcome, including GRADE assessment, and found no difference between the hyperbaric group and the control group. ${ }^{4866}$ No former review comparing adjunctive hyperbaric oxygen therapy with standard care has reported serious adverse events as defined by the The International Council for Harmonisation of Technical Requirements for Pharmaceuticals for Human Use - Good Clinical Practice (ICH-GCP) guidelines.

Quality of life has been poorly addressed in previous literature. Only two randomised clinical trials have dealt with this topic using two different self-reporting questionnaires. ${ }^{2674}$ The results of these two trials has been summarised in eight reviews. ${ }^{5265666972-7575-77}$ The Ontario health assessment found inconsistent results for quality of life, with moderate quality of evidence according to the grade criteria, ${ }^{66}$ whereas Huang et al did not find data available to conduct a meta-analysis. ${ }^{52}$

All the reviews, except one reported data on amputation. ${ }^{31} 485265-6769-737677$ Two reviews found that hyperbaric oxygen therapy significantly reduces the risk of major amputations in persons with diabetic foot ulcers, ${ }^{69} 71$ which was supported in another review without statistical strength. ${ }^{75}$ Goldman found a high level of evidence that hyperbaric oxygen therapy decreases the risk of amputation for patients with diabetic foot ulcers complicated by surgical infection. ${ }^{67}$ Huang et al looked at different diabetic foot ulcer patient groups and found that hyperbaric oxygen therapy was beneficial in preventing amputation in patients with Wagner grade 3 or greater diabetic foot ulcers, who have just undergone surgical debridement of the foot or who had not shown significant improvement in wound healing after 30 or more days of treatment. ${ }^{52}$ The two most recent reviews concluded that there was no statistically significant reduction in major or minor amputation rate with the application of hyperbaric oxygen therapy. ${ }^{48} 72$ The remaining of the reviews were inconclusive regarding hyperbaric oxygen therapy's effect on prevention of amputation due to heterogeneity of data. ${ }^{31} 656670737576$

Many of the trials include Wagner grade 3 that are characterised as deep ulcers with abscesses or osteomyelitis. However, few reviews analyse hyperbaric oxygen therapy's effect on infection in foot ulcers. ${ }^{48267}$ Two former reviews found that hyperbaric oxygen therapy reduced infection in diabetic foot ulcers, ${ }^{52} 69$ with very low quality of evidence. ${ }^{52}$

As a research outcome, diabetic foot ulcer healing is defined in various ways throughout the clinical trials, resulting in a high degree of heterogenicity of this outcome measure. The Ontario health assessment report has for the same reason resulted in low quality of evidence on foot ulcer heling according to the GRADE criteria. ${ }^{66}$ Seven reviews found an effect on ulcer healing in favour of hyperbaric oxygen therapy. ${ }^{52666769707273}$ Three reviews found no difference in wound healing when comparing the effect of hyperbaric oxygen therapy with standard care. $^{486571}$ No review has found an effect on wound healing in favour of standard care as opposed to standard wound care with adjunctive hyperbaric oxygen, and five reviews found insufficient evidence to address this outcome. ${ }^{316875-77}$

It is remarkable that to a wide extent the reviews published between 2000 and 2017 has included the same 2 to 10 randomised clinical trials and several observational studies, with the same six randomised clinical trials occurring in all reviews performed after 2014. Nevertheless, there are great discrepancies in the outcomes of interest and the previous reviews have considerable methodological limitations.

The Cochrane review performed by Kranke et al was the only study with a protocol published ahead of the review. ${ }^{72}$ Only five reviews were based solely on randomised clinical trials, ${ }^{4870-73}$ and two of these are published before 2015 and has therefore not included the most recent trials. ${ }^{70} 78$ The two most recent review that is based solely on randomised clinical trials ${ }^{4873}$ has not included four trials that we have identified after a preliminary search. ${ }^{58-81}$ Three reviews Kranke et al 2015, Health Quality Ontario 2017 and Huang et al 2015 have assigned levels of evidence according to the GRADE approach. ${ }^{82-84}$ However, no former review has taken into account both risks of systematic error and risks of random errors (Cochrane Methodology and Trial Sequential Analysis).

There is a need of an updated systematic review including all relevant trials taking into account risks of design errors, random errors and systematic errors. ${ }^{82}$

\section{Objective}

To assess the beneficial and harmful effects of adding hyperbaric oxygen therapy to standard wound care for diabetic foot ulcers.

\section{METHODS AND ANALYSIS}

The method applied in this systematic review is based on Preferred Reporting Items for Systematic Reviews and Meta-Analysis Protocols (PRISMA-P) guidelines for reporting systematic reviews evaluating healthcare interventions. ${ }^{856}$ The method has previously been applied by our group in systematic reviews with meta-analysis on a wide range of healthcare topics. ${ }^{87-94}$ A PRISMA-P checklist file is attached (online supplementary additional file $1)$.

\section{Criteria for considering studies for this review}

Types of studies

Randomised clinical trials. Trials will be considered irrespective of trial design, setting, publication status, publication year and publication language.

\section{Types of participants}

Persons with diabetic foot ulcers. We will accept the definitions used by the trialists. Persons with diabetic 
foot ulcers will be included irrespective of age, sex and comorbidities.

\section{Types of interventions}

\section{Hyperbaric oxygen therapy group}

We will accept interventions with hyperbaric oxygen therapy in the treatment of diabetic foot ulcer. We will accept the definitions used by the trialists.

\section{Standard wound care}

We will primarily include trials if the trialists plan to deliver a similar type of wound care in both the experiment and control group. We will accept any intervention defined as standard wound care or similar, that is, any intervention where the setting is described as multidisciplinary and where the overall aim is to facilitate healing of a diabetic foot ulcer. Treatment elements of the standard wound care could be offloading, debridement, treatment of infection, vascular assessment, metabolic control and treatment of comorbidities.

\section{Control group}

In addition to standard wound care we will accept if the trialists in the control group deliver a sham intervention. We will accept any definition of sham to hyperbaric oxygen therapy, that is, any regimen where the overall aim is to simulate hyperbaric oxygen therapy. Treatment elements of sham therapy could be dose of intervention; atmospheric pressure and inspiratory partial pressure of oxygen, duration of treatment sessions, number of air-breaks, duration of air-breaks, frequency of air-breaks, number of completed treatment sessions, number of consecutive treatment sessions and duration of treatment breaks.

We will also accept if the trialist delivers other types of co-interventions to standard wound care than hyperbaric oxygen therapy, if the trial compares wound care regimens that included hyperbaric oxygen therapy with wound care regimens that excluded hyperbaric oxygen therapy. If co-interventions differed significantly between trials we will clearly state this and discuss the implications.

\section{Primary outcomes}

1. All-cause mortality.

2. Serious adverse events. We will define a serious adverse event as any untoward medical occurrence that resulted in death, was life-threatening, required hospitalisation or prolongation of existing hospitalisation and resulted in persistent or significant disability or jeopardised the patient. ${ }^{95}$ As we expect the trialists' reporting of serious adverse events to be heterogeneous and not strictly according to the ICH-GCP recommendations, we will include the event as a serious adverse if the trialists either: (1) use the term 'serious adverse event' but does not refer to ICH-GCP or (2) report the proportion of participants with an event that we consider will fulfil the ICH-GCP definition (eg, myocardial infarction or hospitalisation). If several of such events are reported then we will choose the highest proportion reported in each trial.
3. Quality of life measured on any valid scale.

\section{Secondary outcomes}

1. Major amputation (as defined by trialists).

2. Healing of index wound (as defined by the trialists).

3. Wound infection (as defined by the trialists).

\section{Exploratory outcomes}

1. Any amputation.

2. Minor amputation.

All outcomes, except quality of life, will be analysed as proportions of participants in each group.

\section{Assessment time points}

We will use the trial results reported at maximal follow-up for all outcomes. However, if the trialists reported results at multiple time points, we will primarily use the results reported at the time point closest to 12 months after intervention start.

\section{Patient and public involvement}

There was no involvement of patients in the methodological design of this protocol. This protocol for a systematic review is based on published randomised controlled trials and does not involve new patient recruitment. It is designed and conducted in accordance with recommendations stated in the Cochrane Handbook for Systematic Reviews of Interventions. We expect to publish the review in a peer-reviewed scientific journal and thereby disseminated to the public. It will not be possible to disseminate the study results to the participants of the original trials.

\section{Search methods for identification of studies}

\section{Electronic searches}

The following databases will be used; Cochrane Central Register of Controlled Trials (CENTRAL), Medical Literature Analysis and Retrieval System Online (MEDLINE), Excerpta Medica database (Embase), Latin American and Caribbean Health Sciences Literature (LILACS), Science Citation Index Expanded on Web of Science and BIOSIS in order to identify relevant trials. The preliminary search strategy for MEDLINE (Ovid) is given in (online supplementary additional file 2). We will search all databases from their inception to the present.

\section{Searching other resources}

The reference lists of relevant publications will be checked for any unidentified randomised trials. Moreover, authors of included studies will be contacted by email and asked for unpublished randomised trial results. Moreover, we will search for ongoing trials on the following platforms:

- ClinicalTrials.gov (www.clinicaltrials.gov)

- Google Scholar (https://scholar.google.dk/)

- The Turning Research into Practice (TRIP) Database (https://www.tripdatabase.com/)

- European Medicines Agency (EMA) (http://www. ema.europa.eu/ema/)

- United States Food and Drug Administration (FDA) ( www.fda.gov) 
- China Food and Drug Administration (CFDA) (http://eng.sfda.gov.cn/WS03/CL0755/)

- Medicines and Healthcare products Regulatory

- Agency (https://www.gov.uk/government/organisations/medicines-and-healthcare-products-regulatoryagency)

- The WHO

- International Clinical Trials Registry Platform (ICTRP) search portal (http://apps.who.int/trialsearch/)

Additionally, conference abstracts from conferences on hyperbaric oxygen therapy and on diabetic foot ulcers will be hand searched for relevant trials. Unpublished and grey literature trials will be considered for the review. A preliminary systematic search was preformed 15 April 2019.

\section{Data collection and analysis}

We will perform the review in accordance with the recommendations of the Cochrane Collaboration. ${ }^{96}$ The analyses will be performed using Review Manager $5^{97}$ and Trial Sequential Analysis. ${ }^{98} 99$ If Review Manager statistical software is inadequate for all analysis, we will use Stata $14^{100}$ when needed.

\section{Selection of studies}

Two authors (NFRH and JV) will screen titles and abstracts independently. All relevant full-text study reports/publications will be retrieved. Two review authors (NFRH and JV) will independently screen the full texts to identify ineligible trials. Reasons for exclusion of trials will be reported. Any disagreement between the review authors will be resolved through discussion or, if required, a third person will be consulted (JCJ). Trial selection will be displayed in an adapted flow diagram as per the PRISMA statement. $^{84}$

\section{Data extraction and management}

Two authors (NFRH and JV) will independently extract data from included trials. Disagreements will be resolved by discussion with a third author (JCJ). We will assess duplicate publications and companion papers of a trial together to evaluate all available data simultaneously (maximise data extraction and correct bias assessment). We will contact the trial authors by email to specify any additional data, which may not have been reported sufficiently in the publication.

\section{Trial characteristics}

Bias risk components (as defined below); trial design (parallel, factorial or crossover); number of intervention arms; length of follow-up; estimation of sample size; inclusion and exclusion criteria.

\section{Participant characteristics and diagnosis}

Number of randomised participants; number of analysed participants; number of participants lost to follow-up/ withdrawals/crossover; compliance with hyperbaric therapy; age range (mean or median); compliance with standards care and sex ratio; foot ulcer classification system and grade of diabetic foot ulcer (according to the grading system used at the choice of the trialist); type of diabetic foot ulcer (neuropathic, non-neuropathic or unspecified); baseline numbers of diabetes mellitus type 1; baseline numbers of diabetes mellitus type 2; baseline numbers of participants with diabetic comorbidities (ie, hypertension, hyperlipidaemia, arteriosclerosis, neuropathy and nephropathy); baseline numbers of smokers; baseline number of participants with previous cerebrovascular incident; baseline number of participants with heart failure; baseline number of participants with previous myocardial infarction; baseline number of participants with chronic kidney disease; baseline number of participants with peripheral vascular disease; baseline number of participants with arterial insufficiency; baseline number of participants with previous diabetic foot ulcer; baseline number of participants in immunosuppressive treatment; baseline number of participants with previous or active cancer; baseline number of participants with abuse of alcohol or elicit substances and baseline number of participants with previous minor or major amputation. We will additionally report the proportion of participants in the compared groups who received specialised wound dressings and previous peripheral revascularisation.

\section{Hyperbaric oxygen therapy characteristics}

Dose of intervention; atmospheric pressure and partial inspiratory pressure of oxygen; duration of treatment sessions; number of air-breaks; duration of air-breaks; number of completed treatment sessions; number of consecutive treatment sessions and duration of treatment breaks (eg, weekends).

\section{Standard wound care characteristics}

Treatment with offloading; orthotist and prosthetist; podiatric care; restoration of perfusion with vascular intervention; infection control with debridement (eg, partial toe or ray amputation, debridement of ulcer with underlying bursa, cicatrix or bone, incision and drainage of deep space abscess or removal of necrotising soft tissue infection) and antibiotics when needed; treatment of cardiovascular disease; treatment of chronic lung disease; treatment of kidney disease; metabolic control including treatment of oedema and malnutrition.

\section{Sham/control intervention characteristics (when applicable)}

Dose of intervention; atmospheric pressure and inspiratory partial pressure of oxygen; duration of treatment sessions; number of air-breaks; duration of air-breaks; number of completed treatment sessions; oxalated or continuous oscillation as sham pressure; number of consecutive treatment sessions and duration of treatment breaks (eg, weekends).

\section{Co-intervention characteristics}

Type of co-intervention; dose of co-intervention; duration of co-intervention and mode of administration. Co-interventions could be maggot therapy, growth factor therapy, collagen products, bioengineered tissue and stem cells. 


\section{Outcomes}

All outcomes listed above will be extracted from each randomised clinical trial. Incomplete or selectively reported outcomes will be identified in accordance with the criteria described below in the sections 'incomplete outcome data' bias domain and 'selective outcome reporting' bias domain.

\section{Notes}

When available, funding of the trial and authors conflicts of interest will be extracted from the included trials.

Inadequately reported outcome data will be described in the 'Characteristics of included studies' table. Two review authors (NFRH and JV) will independently transfer data into the Review Manager file. ${ }^{86}$ Any disagreement between the review authors will be resolved through discussion and a third person will be consulted when required (JCJ).

\section{Assessment of risk of bias in included studies}

The trial methodology of the included randomised trials will be evaluated according to the instructions specified in the Cochrane Handbook for Systematic Reviews of Interventions, ${ }^{96}$ including assessment of the risk of bias. Furthermore, the methodology will be evaluated in respect to the following criteria:

- Random sequence generation.

- Allocation concealment.

- Blinding of participants and treatment providers.

- Blinding of outcome assessment.

- Incomplete outcome data.

- Selective outcome reporting.

- For profit bias.

- Overall risk of bias.

This allows for classification of randomised trials into low risk of bias and high risk of bias. High risk of bias carries a risk of overestimation of positive intervention effects and underestimations of negative effects. ${ }^{101-107}$

\section{Random sequence generation}

- Low risk: Sequence generation was achieved using a random number table or a computer random number generator. Drawing lots, tossing a coin, shuffling cards and throwing dice will also be considered if it was adequately performed by an independent adjudicator.

- Unclear risk: The trial was presented as being randomised, but the method of randomisation was not specified.

- High risk: The allocation sequence was not randomised or only quasi-randomised. These trials will be excluded.

\section{Allocation concealment}

- Low risk: The allocation of trial participants was performed by a central independent unit, including; on-site locked computer, identical-looking numbered sealed envelopes, drug bottles or containers prepared by an independent investigator.
- Uncertain risk: The allocation concealment process was not described, but the trial was characterised as randomised.

- High risk: The allocation sequence was familiar to the investigators who assigned the participants.

\section{Blinding of participants and treatment providers}

- Low risk: The participants and the treatment providers were blinded to intervention allocation and the blinding procedure was described comprehensively in the trial paper.

- Uncertain risk: The procedure of blinding was insufficiently described or otherwise unclear, but the trial was characterised as blinded.

- High risk: Blinding of participants and the treatment providers was not performed.

\section{Blinding of outcome assessment}

- Low risk of bias: Outcome assessors were blinded and the blinding procedure was described comprehensively in the trial paper.

- Uncertain risk of bias: Blinding of outcome assessors was not performed or described incomprehensively.

- High risk of bias: No blinding had occurred or the blinding of outcome assessors was inadequate.

\section{Incomplete outcome data}

- Low risk of bias: No dropouts or withdrawals in any of the outcomes occurred or missing data were unlikely to bias treatment effects, because numbers and reasons for withdrawals and dropouts were similar in both groups and this was clearly described. Largely, the trial is judged to have low risk of bias due to incomplete outcome data if dropouts were less than $5 \%$. However, the $5 \%$ cut-off is not definitive.

- Uncertain risk of bias: There was insufficient information on missing data to assess the bias on the results.

- High risk of bias: The results were likely to be biassed due to missing data. This could be caused by the different pattern of dropouts in the two groups, or by improper methods when dealing with missing data, for example, last observation carried forward.

\section{Selective outcome reporting}

- Low risk of bias: A protocol was published before or at the time the trial was initiated, and the outcomes described in the protocol were similar to those reported in the trial paper. If all-cause mortality and serious adverse events were reported outcomes in a trial, the trial will be categorised with a grade of low risk of bias, even if the trial protocol was absent or published after initiation of the trial.

- Uncertain risk of bias: A trial protocol was not published and all-cause mortality and serious adverse events were not reported.

- High risk of bias: The outcomes described in the protocol were different from those reported in the trial. 
For profit bias

- Low risk of bias: The trial appeared to be free of other components of for-profit bias.

- Unclear risk of bias: It was unclear whether the trial was free of for-profit bias.

- High risk of bias: There was a high risk of for-profit bias.

\section{Other risks of bias}

- Low risk of bias: The trial appears to be free of other components that could put it at risk of bias, for example, academic bias or for-profit bias.

- Unclear risk of bias: It is uncertain whether the trial is free of other components that could put it at risk of bias.

- High risk of bias: The trial is at risk of bias due to other factors, for example, authors conducted trials on the same topic or for-profit bias.

\section{Overall risk of bias}

- Low risk of bias: When the trial is designated with a 'low risk of bias' with regard to all the bias domains described in the sections above then the trial will be categorised with an overall 'low risk of bias'.

- High risk of bias: If any of the bias risk domains described in the sections above are categorised as 'unclear' or 'high risk of bias' then the trial will be categorised as 'high risk of bias'

In addition to judging the overall risk of bias of each trial, we will assess the bias risk for each outcome by evaluations of the risk domains 'blinding of outcome assessment', 'incomplete outcome data' and 'selective outcome reporting'. Our primary conclusions will be based on our primary outcome results with overall low risk of bias. Both our primary and secondary conclusions will be presented in the summary of findings tables.

\section{Differences between the protocol and the review}

The review will be conducted in consonance with this protocol and any deviations from the protocol will be stated in the section on 'Differences between the protocol and the review' in the systematic review.

\section{Measures of treatment effect}

\section{Dichotomous outcomes}

Risk ratios, with $95 \%$ CI, for dichotomous outcomes and CIs adjusted by the Trial Sequential Analysis (see below) will be calculated.

\section{Continuous outcomes}

The mean differences and the standardised mean difference with 95\% CI for continuous outcomes and CIs adjusted in the Trial Sequential Analysis (see below) will be calculated.

\section{Dealing with missing data}

All trial authors will be contacted to obtain missing data as described above (ie, data extraction and assessment of risk of bias).

\section{Dichotomous outcomes}

Missing values will not be imputed for any outcomes in our primary analysis. In our sensitivity analysis we will impute data (see below).

\section{Continuous outcomes}

Outcome scores will primarily be assessed at single time points. In case that only changes from baseline scores were reported, then we will analyse the results together with follow-up scores. ${ }^{88}$ If SDs were not reported we will use trial data to calculate the SDs, whenever possible. We will only use intention-to-treat data when the randomised trial contains and report such data. Missing values will not be imputed for any outcomes in our primary analysis. However, in the sensitivity analysis for continuous outcomes, data will be imputed (see below).

\section{Assessment of heterogeneity}

Assessment of heterogeneity will primarily be inspected using forest plots. Second, heterogeneity will be statistically evaluated by $\chi^{2}$ test (threshold $\mathrm{p}<0.10$ ) and quantitatively by the $\mathrm{I}^{2}$ statistic. ${ }^{108} 109$

Furthermore, possible heterogeneity will be revealed through subgroup analyses. Ultimately, we may decide that a meta-analysis should be avoided. ${ }^{96}$

\section{Assessment of reporting biases}

If 10 or more randomised trials are included in the review, then we will visually inspect funnel plots to assess the risk of bias. We are aware of the limitations of a funnel plot (ie, a funnel plot needs a range of studies with varying size and subjective judgement is involved) and this will be integrated when assessing possible bias. For dichotomous outcomes, we will test asymmetry with the Harbord test if $\tau^{2}$ is less than 0.1 and with the Rücker test if $\tau^{2}$ is more than $0.1{ }^{110}$ For continuous outcomes, we will use the regression asymmetry test ${ }^{109}$ and the adjusted rank correlation. $^{111}$

\section{Unit of analysis issues}

Only randomised clinical trials will be included in the review, and for trials using crossover design, only data from the first period will be included. ${ }^{96112}$ We will not include cluster randomised trials. Therefore, there will not be any unit of analysis issues.

\section{Data synthesis}

Meta-analysis

We will undertake this meta-analysis according to the recommendations stated in the Cochrane Handbook for Systematic Reviews of Interventions ${ }^{96}$ and Keus et al, ${ }^{84}$ as well as the eight-step assessment suggested by Jakobsen $e t$ $a l .{ }^{82}$ The statistical software Review Manager 5.3 provided by Cochrane will be used to analyse data. ${ }^{97}$

We will apply both random-effects meta-analyses, ${ }^{113}$ and fixed-effects meta-analyses in the assessment of our intervention effects, ${ }^{114}$ and the most conservative point estimate will be used. ${ }^{82}$ The most conservative point estimate is the estimate closest to null effect. If the two estimates 
are similar, we will use the estimate with the widest CI. Our review evaluates a total of six outcomes (primary and secondary), and we will therefore consider a $p$ value of 0.014 or less as our threshold for statistical significance. ${ }^{82}$ We will use the eight-step procedure to evaluate if thresholds for significance are crossed. ${ }^{82}$

In case that multiple trial arms are reported in a single trial, we will only include the relevant arms. If two comparisons are combined in the same meta-analysis, we will halve the number of participants in the control group to avoid double-counting. ${ }^{96}$ Trials with a factorial design will be included. In a $2 \times 2$ factorial designed trial, the two groups receiving standard wound care interventions will be designated standard wound care groups, while the two groups receiving standard wound care with adjunctive hyperbaric oxygen therapy interventions will be designated standard wound care with adjunctive hyperbaric oxygen therapy. If quantitative synthesis is not appropriate, we will report the results in a narrative way.

\section{Trial sequential analysis}

Traditional meta-analysis runs the risk of random errors due to sparse data and repetitive testing of accumulating data when updating reviews. We wish to control the risks of type I errors and type II errors. Therefore, for all outcomes we will perform a Trial Sequential Analysis to calculate the required information size (ie, the number of participants needed in a meta-analysis to detect or reject a certain intervention effect), and the cumulative Z-curve's breach of relevant trial sequential to monitor boundaries. ${ }^{83} 9899$ 115-121 A more detailed description of Trial Sequential Analysis can be found in the Trial Sequential Analysis manual and at http://www.ctu.dk/ tsa/. ${ }^{99}$

For dichotomous outcomes, we will determine the required information size based on the following estimations:

- The observed proportion of participants with an outcome in the control group (ie, the cumulative proportion of participants with an event in the control groups relative to all participants in the control groups).

- A relative risk reduction of $15 \%$.

- An alpha of $1.4 \%$ for our primary outcomes.

- An alpha of $3.3 \%$ for our secondary outcomes.

- A beta of $10 \%$ and diversity as suggested by the trials in the meta-analysis.

For continuous outcomes, we will determine the required information size based on:

- The observed SD in the Trial Sequential Analysis.

- A mean difference of the observed SD/2.

- An alpha of $2.5 \%$ for our primary outcomes.

- An alpha of $3.3 \%$ for our secondary outcomes.

- A beta of $10 \%$.

Subgroup analysis and investigation of heterogeneity

We will perform the following subgroup analysis when analysing the primary outcomes (all-cause mortality, serious adverse event and quality of life).
1. High risk of bias trials compared with low risk of bias trials.

2. Comparison of contemporary clinical hyperbaric oxygen therapy protocol with other hyperbaric oxygen interventions. The contemporary clinical hyperbaric oxygen therapy protocol is defined as breathing $100 \%$ oxygen for $\geq 60 \mathrm{~min}$ at $\geq 2$ atmosphere absolute, for a total of minimum 30 sessions, administered over a period of no longer than 8 weeks. If 40 sessions, then the total treatment duration should be maximum 10 weeks. If air-breaks, then a minimum 20 min between each air-break.

3. Comparison of different wound grades according to the Wagner classification. ${ }^{1-5}$

4. Comparison of age of participants: 0 to 59 years, 60 to 79 years and above 80 years.

5. Comparison of active smokers to non-smokers (as defined by the trialist).

6. Comparison of participants with known peripheral vascular disease to participants without peripheral vascular disease (as defined by the trialist).

7. Comparison of participants with previous extremity amputation (at any level) to participants without previous amputation (as defined by the trialist).

8. Comparison of participants with previous myocardial infarction to participants without previous myocardial infarction (as defined by the trialist).

9. Comparison of participants with chronic kidney disease to participants without chronic kidney disease (as defined by the trialist).

10. Comparison of change in ulcer area (as defined by the trialist).

We will use the formal test for subgroup interactions in Review Manager. ${ }^{97}$

\section{Sensitivity analysis}

To assess the potential impact of the missing data for dichotomous outcomes, we will perform the following sensitivity analyses on both the primary and secondary outcomes:

- 'Best-worst-case' scenario: We will assume that all participants lost to follow-up in the standard wound care with adjunctive hyperbaric oxygen group have survived, had no serious adverse event, had complete healing of index wound, had no amputation and had no infection and that all those participants lost to follow-up in the standard wound care group have not survived, had a serious adverse event, had an unhealed index wound, had an amputation and had an infection.

- 'Worst-best-case' scenario: We will assume that all participants lost to follow-up in the standard wound care with adjunctive hyperbaric oxygen group have not survived, had a serious adverse event, had an unhealed index wound, had an amputation and had infection and that all those participants lost to follow-up in the standard wound care group have survived, had no serious adverse event, had complete 
healing of the index wound, had no amputation and had no infection.

We will present results of both scenarios in our review. When analysing quality of life, a 'beneficial outcome' will be the group mean plus two SDs of the group mean. A 'harmful outcome' will be the group mean minus two SDs of the group mean. We will again use one SD in another sensitivity analysis. ${ }^{82}$

To assess the potential impact of missing SDs for continuous outcomes we will perform the following sensitivity analysis; where SDs are missing and it is not possible to calculate them, we will impute SDs from trials with similar populations and low risk of bias. If we do not find such trials, we will impute SDs from trials with a similar population or we will impute SDs from all trials as a final option. Results of this scenario will be presented in our review.

Other post hoc sensitivity analyses might be warranted if unexpected clinical or statistical heterogeneity is identified during the analysis of the review results. ${ }^{82}$

\section{'Summary of Findings' table}

We will create a Summary of Findings table with each outcome (all-cause mortality, serious adverse event, quality of life, amputation, infection and healing of index wound). First, we will present the results from the trials with low risk of bias. Second, we will present the results on all trials.

We will use the five GRADE considerations (bias risk of the trials, consistency of effect, imprecision, indirectness and publication bias) to assess the quality of the body of evidence that provides data to our meta-analyses. ${ }^{8299}$ 122-124 'Imprecision' will be assessed using Trial Sequential Analysis. ${ }^{82}$ All methodology will be conducted according to recommendations described in Chapter 8 (Section 8.5) and Chapter 12 of the Cochrane Handbook for Systematic Reviews of Interventions using GRADE pro software ${ }^{96}$ Whenever necessary, we will support all decisions to downgrade the quality of trials with clear arguments in footnotes, in order to aid the reader's understanding of the table and the process.

\section{Current status the study}

Not initiated.

\section{ETHICS AND DISSEMINATION}

As this is a protocol for a systematic review, we use publicly accessible documents as evidence and there is no participant involvement at an individual level, the anonymity of research participants will not be jeopardised and an institutional ethics approval is not required. We will seek to publish the results of the review in a peer-reviewed academic research paper, also in the event of insignificant results or null results and thereby it will be disseminated to clinicians and public available.

\section{DISCUSSION}

This protocol aims at assessing the beneficial and harmful effects of adding hyperbaric oxygen therapy to standard wound care in the treatment of diabetic foot ulcers. The outcomes assessed with this protocol will be; all-cause mortality, serious adverse events, quality of life, healing of the index foot ulcer, major amputation and wound infection. These outcomes are meaningful, valuable and helpful to persons with diabetic foot ulcers and provides evidence on the effectiveness, benefits and harms of hyperbaric oxygen therapy. We accept trials with any definition of diabetic foot ulcers, and irrespective of participants age, sex and comorbidities, and thereby minimise discrimination and attempt to reduce risks of research only benefitting specific groups. We assess the quality of evidence and the strength of our recommendations through the GRADE approach and thereby stipulates informed healthcare decisions for use in clinical everyday practice.

The limitations of this study are mostly related to multiple testing and a heterogeneous data set. We minimise the limitations of pooling the results from a heterogeneous population, by systematically assessing both statistical and clinical heterogeneity and we have planned several subgroup and sensitivity analyses. Major amputation is assessed as a secondary outcome and minor amputation as well as any amputation are assessed as exploratory outcomes. The definition of minor and major amputation may vary across trials. Moreover, if the trials are not adequately blinded, the decision to perform a major or minor amputation in a given participant may be biassed. We plan to thoroughly relate the results of 'major amputation' to both the results of 'any amputation' and 'minor amputation' when interpreting the review results. The Wagner classification system for grading diabetic foot ulcers is based on the depth of penetration, the presence of osteomyelitis or gangrene and the extent of tissue necrosis. The drawback of the Wagner classification system is that it does not specifically address critically important parameters such as ischaemia and infection and is therefore by many considered outdated and inappropriate to assess the severity of a diabetic foot ulcer as discussed above. Nevertheless, we used the Wagner classification to grade the ulcers in our subgroup analysis. Our preliminary search on the trials included in this review showed that they did not provide enough details to describe the wound by any other classification system. The importance of ischaemia and infection will be revealed through separate analysis. We have adjusted our threshold for significance according to the number of primary outcomes. However, the number of subgroup analysis also adds to the risk of type 1 error, which will be considered when interpreting the review results.

If this protocol is followed the possible impact on organisations could be change of guideline for treatment of diabetic foot ulcers and thereby possibly benefit numerous persons with diabetic foot ulcers. 


\section{Author affiliations}

${ }^{1}$ Department of Anaesthesia, Centre of Head and Orthopaedics, Copenhagen University Hospital, Copenhagen, Rigshospitalet, Denmark

${ }^{2}$ The Copenhagen Trial Unit, Centre for Clinical Intervention Research, Copenhagen University Hospital, Copenhagen, Rigshospitalet, Denmark

${ }^{3}$ Department of Cardiology, Holbaek Sygehus, Holbaek, Sjaelland, Denmark

${ }^{4}$ Department of Regional Health Research, The Faculty of Heath Sciences University of Southern Denmark, Odense, Denmark

${ }^{5}$ Department of Cardiology, Holbæk Hospital, Holbaek, Denmark

Contributors JV drafted the protocol. NFRH, JCJ and $\mathrm{OH}$ amended the protocol. All authors read and approved the final manuscript.

Funding The authors have not declared a specific grant for this research from any funding agency in the public, commercial or not-for-profit sectors.

Competing interests None declared.

Patient and public involvement Patients and/or the public were not involved in the design, or conduct, or reporting or dissemination plans of this research.

Patient consent for publication Not required

Provenance and peer review Not commissioned; externally peer reviewed.

Open access This is an open access article distributed in accordance with the Creative Commons Attribution Non Commercial (CC BY-NC 4.0) license, which permits others to distribute, remix, adapt, build upon this work non-commercially, and license their derivative works on different terms, provided the original work is properly cited, appropriate credit is given, any changes made indicated, and the use is non-commercial. See: http://creativecommons.org/licenses/by-nc/4.0/.

\section{ORCID iD}

Niels Frederich Rose Holm http://orcid.org/0000-0002-2289-272X

\section{REFERENCES}

1 Boulton AJM, Vileikyte L, Ragnarson-Tennvall G, et al. The global burden of diabetic foot disease. Lancet 2005:366:1719-24.

2 World Health Organization. Global report on diabetes. World Health organization., 2016. Available: http://www.who.int/iris/handle/ 10665/204871 [Accessed 10 Apr 2019].

3 Singh N, Armstrong DG, Lipsky BA. Preventing foot ulcers in patients with diabetes. JAMA 2005;293:217-28.

4 Jeffcoate WJ, Harding KG, ulcers Dfoot. Diabetic foot ulcers. The Lancet 2003:361:1545-51.

5 Alexiadou K, Doupis J. Management of diabetic foot ulcers. Diabetes Ther 2012;3:4.

6 Hinchliffe RJ, Brownrigg JRW, Apelqvist J, et al. IWGDF guidance on the diagnosis, prognosis and management of peripheral artery disease in patients with foot ulcers in diabetes. Diabetes Metab Res Rev 2016;32 Suppl 1:37-44.

7 Apelqvist J, Bakker K, van Houtum WH, et al. International Working group on the diabetic foot editorial B. practical guidelines on the management and prevention of the diabetic foot: based upon the International consensus on the diabetic foot (2007). Diabetes Metab Res Rev 2008;24:S181-7.

8 Gore M, Brandenburg NA, Dukes E, et al. Pain severity in diabetic peripheral neuropathy is associated with patient functioning, symptom levels of anxiety and depression, and sleep. J Pain Symptom Manage 2005;30:374-85.

9 Zelman DC, Brandenburg NA, Gore M. Sleep impairment in patients with painful diabetic peripheral neuropathy. Clin J Pain 2006;22:681-5.

10 Galer BS, Gianas A, Jensen MP. Painful diabetic polyneuropathy: epidemiology, pain description, and quality of life. Diabetes Res Clin Pract 2000;47:123-8.

11 Lalli P, Chan A, Garven A, et al. Increased gait variability in diabetes mellitus patients with neuropathic pain. $J$ Diabetes Complications 2013;27:248-54.

12 Nikoloudi M, Eleftheriadou I, Tentolouris A, et al. Diabetic foot infections: update on management. Curr Infect Dis Rep 2018;20:40.

13 Lipsky BA, Aragón-Sánchez J, Diggle M, et al. IWGDF guidance on the diagnosis and management of foot infections in persons with diabetes. Diabetes Metab Res Rev 2016;32 Suppl 1:45-74.

14 Digital NHS. National Diabetes Foot Care Audit, Hospital Admissions Report 2014-2016. V1.0. England and Wales, 2017. https://files.digital.nhs.uk/publication/0/b/ndfa_2014-2016 hospital_admissions_report.pdf
15 Armstrong DG, Lavery LA, Harkless LB. Validation of a diabetic wound classification system. The contribution of depth, infection, and ischemia to risk of amputation. Diabetes Care 1998;21:855-9.

16 Nazri MY, Aminudin CA, Ahmad FS, et al. Quality of life of diabetes amputees following major and minor lower limb amputations. Med $J$ Malaysia 2019;74:25-9.

17 Bhuvaneswar CG, Epstein LA, Stern TA. Reactions to amputation: recognition and treatment. Prim Care Companion J Clin Psychiatry 2007;9:303-8.

18 Tchero $\mathrm{H}$, Kangambega $\mathrm{P}$, Lin L, et al. Cost of diabetic foot in France, Spain, Italy, Germany and United Kingdom: a systematic review. Ann Endocrinol 2018;79:67-74.

19 Prompers L, Huijberts M, Schaper N, et al. Resource utilisation and costs associated with the treatment of diabetic foot ulcers. prospective data from the Eurodiale study. Diabetologia 2008; $51: 1826-34$

20 Hicks CW, Selvarajah S, Mathioudakis N, et al. Burden of Infected Diabetic Foot Ulcers on Hospital Admissions and Costs. Ann Vasc Surg 2016;33:149-58.

21 Bakker K, Apelqvist J, Lipsky BA, et al. The 2015 IWGDF quidance on the prevention and management of foot problems in diabetes. Int Wound J 2016;13:1072.

22 Jeon B-J, Choi HJ, Kang JS, et al. Comparison of five systems of classification of diabetic foot ulcers and predictive factors for amputation. Int Wound J 2017:14:537-45.

23 Bravo-Molina A, Linares-Palomino JP, Vera-Arroyo B, et al. InterObserver agreement of the wagner, University of Texas and pedis classification systems for the diabetic foot syndrome. Foot Ankle Surg 2018;24:60-4.

24 Game F. Classification of diabetic foot ulcers. Diabetes Metab Res Rev 2016;32 Suppl 1:186-94.

25 Ghotaslou R, Memar MY, Alizadeh N. Classification, microbiology and treatment of diabetic foot infections. J Wound Care 2018:27:434-41.

26 Löndahl M, Katzman P, Nilsson A, et al. Hyperbaric oxygen therapy facilitates healing of chronic foot ulcers in patients with diabetes. Diabetes Care 2010;33:998-1003

27 Lavery LA, Armstrong DG, Murdoch DP, et al. Validation of the infectious diseases Society of America's diabetic foot infection classification system. Clin Infect Dis 2007;44:562-5.

28 Widatalla AH, Mahadi SEI, Shawer MA, et al. Implementation of diabetic foot ulcer classification system for research purposes to predict lower extremity amputation. Int J Diabetes Dev Ctries 2009;29:1-5.

29 Schaper NC. Diabetic foot ulcer classification system for research purposes: a progress report on criteria for including patients in research studies. Diabetes Metab Res Rev 2004;20 Suppl 1:S90-5.

30 Gibbons GW, Shaw PM. Diabetic vascular disease: characteristics of vascular disease unique to the diabetic patient. Semin Vasc Surg 2012;25:89-92.

31 Game FL, Hinchliffe RJ, Apelqvist J, et al. A systematic review of interventions to enhance the healing of chronic ulcers of the foot in diabetes. Diabetes Metab Res Rev 2012;28 Suppl 1:119-41.

32 Hinchliffe RJ, Andros G, Apelqvist J, et al. A systematic review of the effectiveness of revascularization of the ulcerated foot in patients with diabetes and peripheral arterial disease. Diabetes Metab Res Rev 2012;28 Suppl 1:179-217.

33 Critchley JA, Carey IM, Harris T, et al. Glycemic control and risk of infections among people with type 1 or type 2 diabetes in a large primary care cohort study. Diabetes Care 2018:41:2127-35.

34 American Diabetes Association. Consensus development conference on diabetic foot wound care: 7-8 April 1999, Boston, Massachusetts. American diabetes association. Diabetes Care 1999;22:1354-60.

35 Bus SA, Armstrong DG, van Deursen RW, et al. IWGDF quidance on footwear and offloading interventions to prevent and heal foot ulcers in patients with diabetes. Diabetes Metab Res Rev 2016;32 Suppl 1:25-36.

36 Bus SA, van Netten JJ, Lavery LA, et al. IWGDF guidance on the prevention of foot ulcers in at-risk patients with diabetes. Diabetes Metab Res Rev 2016;32 Suppl 1:16-24.

37 Game FL, Attinger C, Hartemann A, et al. IWGDF guidance on use of interventions to enhance the healing of chronic ulcers of the foot in diabetes. Diabetes Metab Res Rev 2016;32 Suppl 1:75-83.

38 Schaper NC, Van Netten JJ, Apelqvist J, et al. Prevention and management of foot problems in diabetes: a summary guidance for daily practice 2015 , based on the IWGDF guidance documents. Diabetes Res Clin Pract 2017;124:84-92.

39 Jacobson JH, Morsch JH, Rendell-Baker L. Clinical experience and implications of hyperbaric oxygenation. The historical perspective of hyperbaric therapy. Ann N Y Acad Sci 1965;117:651-70. 
40 Brown IW, Fuson RL, Mauney FM, et al. Hyperbaric oxygenation (hybaroxia): current status, possiblities and limitations. Adv Surg 1965;1:285-349.

41 Weaver LK. Hyperbaric oxygen therapy indications. Thirteenth edn. North Palm Beach, Florida 33408: Best Publishing Company, 2014.

42 Thom SR. Hyperbaric oxygen: its mechanisms and efficacy. Plast Reconstr Surg 2011;127 Suppl 1:131S-41.

43 Boerema I, Meyne NG, Brummelkamp WH, et al. [Life without blood]. Ned Tijdschr Geneeskd 1960;104:949-54.

44 Fife CE, Buyukcakir C, Otto $\mathrm{GH}$, et al. The predictive value of transcutaneous oxygen tension measurement in diabetic lower extremity ulcers treated with hyperbaric oxygen therapy: a retrospective analysis of 1,144 patients. Wound Repair Regen 2002;10:198-207.

45 Hopf HW, Kelly M, Shapshak D. Oxygen and the Basic Mechanisms of Wound Healing.. In: Physiology and medicine of hyperbaric oxygen therapy. 1st ed. Philadelphia, PA, USA: Saunders Elsevier, 2008: 203-28.

46 Rollins MD, Gibson JJ, Hunt TK, et al. Wound oxygen levels during hyperbaric oxygen treatment in healing wounds. Undersea Hyperb Med 2006;33:17-25.

47 Camporesi EM. Side effects of hyperbaric oxygen therapy. Undersea Hyperb Med 2014;41:253-7.

48 Zhao D, Luo S, Xu W, et al. Efficacy and safety of hyperbaric oxygen therapy used in patients with diabetic foot: a meta-analysis of randomized clinical trials. Clin Ther 2017;39:2088-94.

49 hadanny A, Meir O, Bechor Y, et al. The safety of hyperbaric oxygen treatment--retrospective analysis in 2,334 patients. Undersea Hyperb Med 2016;43:113-22.

50 Chuck AW, Hailey D, Jacobs P, et al. Cost-Effectiveness and budget impact of adjunctive hyperbaric oxygen therapy for diabetic foot ulcers. Int J Technol Assess Health Care 2008;24:178-83.

51 Guo S, Counte MA, Gillespie KN, et al. Cost-Effectiveness of adjunctive hyperbaric oxygen in the treatment of diabetic ulcers. Int J Technol Assess Health Care 2003;19:731-7.

52 Huang ET, Mansouri J, Murad $\mathrm{MH}$, et al. A clinical practice guideline for the use of hyperbaric oxygen therapy in the treatment of diabetic foot ulcers. Undersea Hyperb Med 2015;42:205-47.

53 Heyboer M, Sharma D, Santiago W, et al. Hyperbaric oxygen therapy: side effects defined and quantified. Adv Wound Care 2017;6:210-24

54 Walsh JW, Hoffstad OJ, Sullivan MO, et al. Association of diabetic foot ulcer and death in a population-based cohort from the United Kingdom. Diabet. Med. 2016;33:1493-8.

55 Karadurmus N, Sahin M, Tasci C, et al. Potential benefits of hyperbaric oxygen therapy on atherosclerosis and glycaemic control in patients with diabetic foot. Endokrynol Pol 2010;61:275-9.

56 Aydin F, Kaya A, Karapinar L, et al. Igf-1 increases with hyperbaric oxygen therapy and promotes wound healing in diabetic foot ulcers. J Diabetes Res 2013:567834.

57 Chen S-Y, Giurini JM, Karchmer AW. Invasive systemic infection after hospital treatment for diabetic foot ulcer: risk of occurrence and effect on survival. Clin Infect Dis 2017;64:326-34.

58 Ashmawy EM, Shehab Mahmoud ABD El- Kader. Impact of different therapeutic modalities on healing of diabetic foot ulcers. Eur J Gen Pract 2015;12:319-25.

59 Fagher K, Nilsson A, Löndahl M. Heart rate-corrected QT interval prolongation as a prognostic marker for 3-year survival in people with type 2 diabetes undergoing above-ankle amputation. Diabet Med 2015:32:679-85.

60 Wang S, He Y, Xu L, et al. Association between QTc interval prolongation and outcomes of diabetic foot ulcers: data from a 4-year follow-up study in China. Diabetes Res Clin Pract 2018;138:26-34.

61 Sun TB, Yang CCH, Kuo TBJ. Effect of hyperbaric oxygen on cardiac neural regulation in diabetic individuals with foot complications. Diabet Med 2006;23:360-6.

62 Carrington AL, Shaw JE, Van Schie CHM, et al. Can motor nerve conduction velocity predict foot problems in diabetic subjects over a 6-year outcome period? Diabetes Care 2002;25:2010-5.

63 Cameron NE, Eaton SE, Cotter MA, et al. Vascular factors and metabolic interactions in the pathogenesis of diabetic neuropathy. Diabetologia 2001;44:1973-88.

64 Malik RA, Masson EA, Sharma AK, et al. Hypoxic neuropathy: relevance to human diabetic neuropathy. Diabetologia 1990;33:311-8.

65 O'Reilly D, Pasricha A, Campbell K, et al. Hyperbaric oxygen therapy for diabetic ulcers: systematic review and meta-analysis. Int J Technol Assess Health Care 2013;29:269-81.
66 Health Quality Ontario. Hyperbaric oxygen therapy for the treatmen of diabetic foot ulcers: a health technology assessment. Ont Health Technol Assess Ser 2017;17:1-142.

67 Goldman RJ. Hyperbaric oxygen therapy for wound healing and limb salvage: a systematic review. $P M \& R$ 2009;1:471-89.

68 Wang C, Schwaitzberg S, Berliner E, et al. Hyperbaric oxygen for treating wounds: a systematic review of the literature. Arch Surg 2003;138:272-9.

69 Liu R, Li L, Yang M, et al. Systematic review of the effectiveness of hyperbaric oxygenation therapy in the management of chronic diabetic foot ulcers. Mayo Clin Proc 2013;88:166-75.

70 Stoekenbroek RM, Santema TB, Legemate DA, et al. Hyperbaric oxygen for the treatment of diabetic foot ulcers: a systematic review. Eur J Vasc Endovasc Surg 2014;47:647-55.

71 Roeckl-Wiedmann I, Bennett M, Kranke P. Systematic review of hyperbaric oxygen in the management of chronic wounds. $\mathrm{Br} \mathrm{J}$ Surg 2005;92:24-32.

72 Kranke P, Bennett MH, Martyn-St James M, et al. Hyperbaric oxygen therapy for chronic wounds. Cochrane Database Syst Rev 2015;6:CD004123

73 Golledge J, Singh TP. Systematic review and meta-analysis of clinical trials examining the effect of hyperbaric oxygen therapy in people with diabetes-related lower limb ulcers. Diabet. Med. 2019;376.

74 Abidia A, Laden G, Kuhan G, et al. The role of hyperbaric oxygen therapy in ischaemic diabetic lower extremity ulcers: a doubleblind randomised-controlled trial. Eur J Vasc Endovasc Surg 2003;25:513-8.

75 Löndahl M. Hyperbaric oxygen therapy as adjunctive treatment for diabetic foot ulcers. Int J Low Extrem Wounds 2013;12:152-7.

76 Bishop AJ, Mudge E. Diabetic foot ulcers treated with hyperbaric oxygen therapy: a review of the literature. Int Wound $J$ 2014;11:28-34

77 Wunderlich RP, Peters EJ, Lavery LA. Systemic hyperbaric oxygen therapy: lower-extremity wound healing and the diabetic foot. Diabetes Care 2000;23:1551-5.

78 Santema KTB, Stoekenbroek RM, Koelemay MJW, et al. Hyperbaric Oxygen Therapy in the Treatment of Ischemic Lower- Extremity Ulcers in Patients With Diabetes: Results of the $\mathrm{DAMO}_{2}$ CLES Multicenter Randomized Clinical Trial. Diabetes Care 2018;41:112-9.

79 Lin TF CS, Niu KC. The vascular effects of hyperbaric oxygen therapy in treatment of early diabetic foot. Undersea Hyperb Med 2001;28

80 Wang C-J, Wu R-W, Yang Y-J. Treatment of diabetic foot ulcers: a comparative study of extracorporeal shockwave therapy and hyperbaric oxygen therapy. Diabetes Res Clin Pract 2011;92:187-93.

81 Kaur S, Pawar M, Banerjee N, et al. Evaluation of the efficacy of hyperbaric oxygen therapy in the management of chronic nonhealing ulcer and role of periwound transcutaneous oximetry as a predictor of wound healing response: a randomized prospective controlled trial. J Anaesthesiol Clin Pharmacol 2012;28:70-5.

82 Jakobsen JC, Wetterslev J, Winkel P, et al. Thresholds for statistical and clinical significance in systematic reviews with meta-analytic methods. BMC Med Res Methodol 2014;14:120.

83 Wetterslev J, Thorlund K, Brok J, et al. Trial sequential analysis may establish when firm evidence is reached in cumulative metaanalysis. J Clin Epidemiol 2008;61:64-75.

84 Keus F, Wetterslev J, Gluud C, et al. Evidence at a glance: error matrix approach for overviewing available evidence. BMC Med Res Methodol 2010;10:90.

85 Moher D, Liberati A, Tetzlaff J, et al. Preferred reporting items for systematic reviews and meta-analyses: the PRISMA statement. Ann Intern Med 2009;151:w64:264-9.

86 Shamseer L, Moher D, Clarke M, et al. Preferred reporting items for systematic review and meta-analysis protocols (PRISMA-P) 2015: elaboration and explanation. BMJ 2015;349:g7647.

87 Barbateskovic M, Larsen LK, Oxenbøll-Collet M, et al. Pharmacological interventions for delirium in intensive care patients: a protocol for an overview of reviews. Syst Rev 2016;5:211.

88 Marker S, Perner A, Wetterslev J, et al. Stress ulcer prophylaxis versus placebo or no prophylaxis in adult hospitalised acutely ill patients-protocol for a systematic review with meta-analysis and trial sequential analysis. Syst Rev 2017;6:118.

89 Sethi NJ, Nielsen EE, Safi S, et al. Digoxin for atrial fibrillation and atrial flutter: a systematic review with meta-analysis and trial sequential analysis of randomised clinical trials. PLoS One 2018;13:e0193924. 
90 Barbateskovic M, Kraus SR, Collet MO, et al. Haloperidol for delirium in critically ill patients - protocol for a systematic review. Acta Anaesthesiol Scand 2018.

91 Sethi N, Naqash Al, Nielsen N, et al. Fever control interventions versus placebo, sham or no intervention in adults: a protocol for a systematic review with meta-analysis and trial sequential analysis. BMJ Open 2019;9:e032389.

92 Juul S, Poulsen S, Lunn S, et al. Short-Term versus long-term psychotherapy for adult psychiatric disorders: a protocol for a systematic review with meta-analysis and trial sequential analysis. Syst Rev 2019;8:169.

93 Maagaard M, Barbateskovic M, Perner A, et al. Dexmedetomidine for the management of delirium in critically ill patients-A protocol for a systematic review. Acta Anaesthesiol Scand 2019;63:549-57.

94 Nielsen EE, Feinberg J, Raymond I, et al. The effects of adding angiotensin receptor neprilysin inhibitors to usual care in patients with heart failure: a protocol for a systematic review of randomised clinical trials with meta-analysis and trial sequential analysis. Syst Rev 2019;8:251.

95 International Conference on harmonisation of technica requirements for registration of pharmaceuticals for human use (ICH) adopts consolidated guideline on good clinical practice in the conduct of clinical trials on medicinal products for human use. Int Dig Health Legis 1997;48:231-4.

96 Higgins JPT, Green S. Cochrane Handbook for systematic reviews of interventions. The Cochrane Collaboration, 2011. www. handbook.cochrane.org

97 The Nordic Cochrane Centre. Cochrane Revman version 5.3, 2014. Available: https://community.cochrane.org/help/tools-and-software/ revman-5

98 CTU. TSA - trial sequential analysisCopenhagen trial unit. Available: http://www.ctu.dk/tsa/ [Accessed 10 Apr 2019].

99 Thorlund K, Engstrøm J, Wetterslev J, et al. User manual for trial sequential analysis (TSA) Copenhagen trial unit, centre for clinical intervention research, Denmark;, 2011. Available: http://www.ctu. $\mathrm{dk} / \mathrm{tsa} / \mathrm{files} / \mathrm{tsa}$ manual.pdf [Accessed $10 \mathrm{Apr} 2019$ ].

100 StataCorp. Stata release 14. statistical software. College Station, tx: StataCorp Lp, 2014. Available: http://www.stata.com [Accessed 10 Apr 2019].

101 Gluud LL. Bias in clinical intervention research. Am J Epidemiol 2006;163:493-501.

102 Kjaergard LL, Villumsen J, Gluud C. Reported methodologic quality and discrepancies between large and small randomized trials in meta-analyses. Ann Intern Med 2001;135:982-9.

103 Lundh A, Sismondo S, Lexchin J, et al. Industry sponsorship and research outcome. Cochrane Database Syst Rev 2012;12:Mr000033.

104 Moher D, Pham B, Jones A, et al. Does quality of reports of randomised trials affect estimates of intervention efficacy reported in meta-analyses? Lancet 1998;352:609-13.

105 Schulz KFet al. Empirical evidence of bias. JAMA 1995;273:408-12.

106 Wood L, Egger M, Gluud LL, et al. Empirical evidence of bias in treatment effect estimates in controlled trials with different interventions and outcomes: meta-epidemiological study. BMJ 2008;336:601-5.
107 Savović J, Jones H, Altman D, et al. Influence of reported study design characteristics on intervention effect estimates from randomised controlled trials: combined analysis of metaepidemiological studies. Health Technol Assess 2012;16:1-82.

108 Higgins JPT, Thompson SG. Quantifying heterogeneity in a metaanalysis. Stat Med 2002;21:1539-58.

109 Higgins JPT, Thompson SG, Deeks JJ, et al. Measuring inconsistency in meta-analyses. BMJ 2003;327:557-60.

110 Harbord RM, Egger M, Sterne JAC. A modified test for small-study effects in meta-analyses of controlled trials with binary endpoints. Stat Med 2006;25:3443-57.

111 Begg CB, Mazumdar M. Operating characteristics of a RANK correlation test for publication bias. Biometrics 1994;50:1088-101.

112 Elbourne DR, Altman DG, Higgins JPT, et al. Meta-Analyses involving cross-over trials: methodological issues. Int J Epidemiol 2002;31:140-9.

113 DerSimonian R, Laird N. Meta-Analysis in clinical trials. Control Clin Trials 1986;7:177-88.

114 Demets DL. Methods for combining randomized clinical trials: strengths and limitations. Stat Med 1987;6:341-8.

115 Brok J, Thorlund K, Gluud C, et al. Trial sequential analysis reveals insufficient information size and potentially false positive results in many meta-analyses. J Clin Epidemiol 2008;61:763-9.

116 Brok J, Thorlund K, Wetterslev J, et al. Apparently conclusive metaanalyses may be inconclusive--Trial sequential analysis adjustment of random error risk due to repetitive testing of accumulating data in apparently conclusive neonatal meta-analyses. Int $\mathrm{J}$ Epidemiol 2009;38:287-98.

117 Thorlund K, Devereaux PJ, Wetterslev J, et al. Can trial sequential monitoring boundaries reduce spurious inferences from metaanalyses? Int J Epidemiol 2009;38:276-86.

118 Wetterslev J, Thorlund K, Brok J, et al. Estimating required information size by quantifying diversity in random-effects model meta-analyses. BMC Med Res Methodol 2009;9:86.

119 Thorlund K, Anema A, Mills E. Interpreting meta-analysis according to the adequacy of sample size. An example using isoniazid chemoprophylaxis for tuberculosis in purified protein derivative negative HIV-infected individuals. Clin Epidemiol 2010;2:57-66.

120 Imberger G, Gluud C, Boylan J, et al. Systematic reviews of anesthesiologic interventions reported as statistically significant: problems with power, precision, and type 1 error protection. Anesth Analg 2015;121:1611-22.

121 Imberger G, Thorlund K, Gluud C, et al. False-Positive findings in Cochrane meta-analyses with and without application of trial sequential analysis: an empirical review. BMJ Open 2016;6:e011890.

122 Guyatt GH, Oxman AD, Vist GE, et al. Grade: an emerging consensus on rating quality of evidence and strength of recommendations. BMJ 2008;336:924-6.

123 Schünemann HJ, Best D, Vist G, et al. Letters, numbers, symbols and words: how to communicate grades of evidence and recommendations. CMAJ 2003;169:677-80.

124 Guyatt GH, Oxman AD, Schünemann HJ, et al. Grade guidelines: a new series of articles in the Journal of clinical epidemiology. $J$ Clin Epidemiol 2011;64:380-2. 